\title{
Deformable Registration of Cortical Structures via Hybrid Volumetric and Surface Warping
}

\author{
Tianming Liu, Dinggang Shen, and Christos Davatzikos \\ Section of Biomedical Image Analysis, Department of Radiology, \\ University of Pennsylvania, Philadelphia, PA 19104 \\ \{tliu, dgshen, christos\}erad.upenn.edu
}

\begin{abstract}
This paper presents a method of deformable registration of cortical structures across individuals, using hybrid volumetric and surface warping. The proposed method uses two steps. In the first step, a HAMMER-based volumetric registration algorithm warps the model surface to the individual's space. In the second step, an attribute-based surface registration method further refines the results of the volumetric warping. An attribute vector is defined for each vertex on the cortical surface, and used to capture the local and global geometric features of the surface patch. The attribute vector is designed to be as distinctive as possible, so that each vertex on the model surface can find its correspondence on the individual surface. Experimental results on synthesized and real brain data are provided to demonstrate the performance of the proposed method in registering cortical structures across individuals.
\end{abstract}

\section{Introduction}

Registration of cortical surfaces across individuals is a very important step for quantitative analysis of brain cortices. However, this is a very difficult task, due to the highly convoluted and highly irregular nature of the cortex, and the large variability of individual cortices. To date, several methods have been proposed to directly register cortical surfaces, using geometric information from these surfaces such as curvature and geodesic features $[1,2]$. As cortical surfaces are very variable, the corresponding geometric features may be inconsistent across individuals. Also, different structures in the same individual, such as the post-central gyrus and the pre-central gyrus, may have very similar geometric features. For these reasons, achieving accurate results with surface-based registration methods has proven difficult.

In this paper, we present a hybrid volumetric and surface warping method for registration of cortical surfaces, by combining the advantages of the volumetric and the surface warping methods. First, the model cortical surface is warped to match the individual's cortical surface using a HAMMER-based volumetric registration algorithm [3]. This procedure greatly removes the variability between the cortical surfaces of the model and the individual, and thus provides a very good initialization for the 
sequent surface-based registration step. Second, the warped model surface is further registered to the individual cortical surface, using an evolution of an attribute-based surface matching technique presented in [4]. An attribute vector of a surface vertex is used to capture local and global geometric features of the cortical surface. The attribute vector is designed to be as distinctive as possible, in order to distinguish the different parts of cortical surface.

\section{Method}

Our cortical surface registration method can be formulated as a warping procedure, $h: S^{\mathbf{M d l}} \rightarrow S^{\text {Sub }}$, where $S^{\text {Mdl }}$ and $S^{\text {Sub }}$ are model and target surfaces, respectively, and $h$ is a transformation. This warping procedure is decomposed into two steps, i.e., $h=h_{2} \cdot h_{1}$. The first transformation $h_{1}$ is obtained by a HAMMER-based volumetric warping step, resulting in a volume-warped model surface $S^{\mathbf{W} \_ \text {Mdl }}$, i.e., $S^{\mathbf{W} \_ \text {Mdl }}=h_{1}\left(S^{\text {Mdl }}\right)$. The second transformation $h_{2}$ is determined by an attributebased surface warping step, which results in a finally warped model surface $S^{\text {Final }}$, i.e., $S^{\text {Final }}=h_{2}\left(S^{\mathbf{W}_{-} \mathbf{M d l}}\right)$. In the following, we will describe the second step of surface-based warping, which involves defining an attribute vector for each vertex and using an attribute-based correspondence detection method for warping cortical surfaces.

\subsection{Attribute Vector}

In the surface warping step, each model vertex should be warped to its corresponding one in the individual. To achieve this, an attribute vector, which we call clamphistogram, is defined for each vertex, and used to capture both local and global geometric features of the surface around the vertex under consideration. The attribute vector is designed to be as distinctive as possible, so that each vertex in the model surface can easily find its corresponding one in the individual surface.

Clamp-histogram is the accumulation of angles between the normal direction of a vertex $v$ and the normal directions of the neighboring vertices $\left\{v_{j} \in P(v, r)\right\}$, where $P(v, r)$ is a surface patch centered on the vertex $v$ and with a geodesic distance of $r$. In this study, we use $r=20 \mathrm{~mm}$. As demonstrated in Fig. 1, the angle between the normal directions of vertex $v$ and the neighboring vertex $v_{j}$ can be calculated by

$$
\alpha_{j}=\cos ^{-1}\left(n(v) \cdot n\left(v_{j}\right)\right),
$$

where $n(\cdot)$ denotes the normal direction of a vertex, and angle $\alpha_{j}$ is in the range of $[0, \pi]$. To calculate the histogram of the angles, the entire angle range $[0, \pi]$ is di- 
vided into $N$ equal segments, so that each segment will count the accumulation of angles in its domain. In this study, we use $N=18$. Let $\{H(v, k), k \in[1, N]\}$ denote the histogram of angles for the vertex $v$; then the vector $A(v)=[H(v, 1), \cdots, H(v, N)]^{T}$ can be used as an attribute vector for vertex $v$, to represent geometric information of the surface patch around vertex $v$. Notably, the attribute vector $A(v)$ is invariant to rigid transformation. Therefore, the attribute vectors can be used to directly detect the correspondences of vertices during the registration process of cortical surfaces.

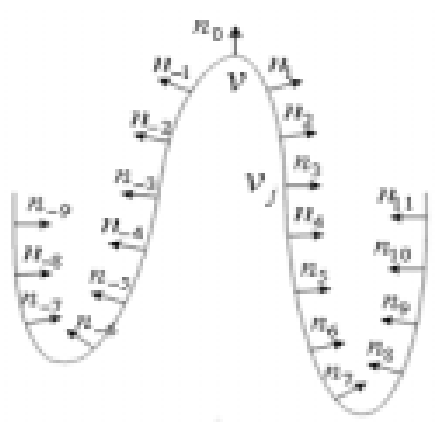

(a)

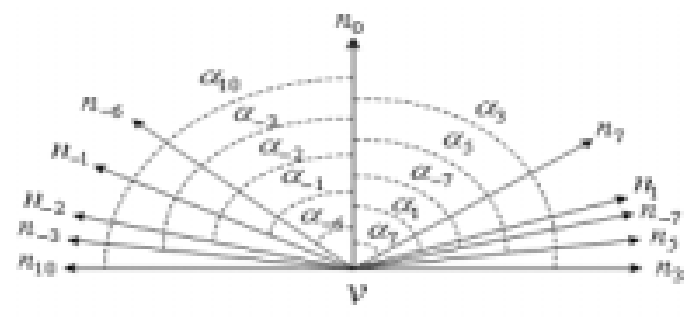

(b)

Fig. 1. Schematic illustration of the clamp-histogram. (a) Normal vectors $n_{i}$ on the vertex under consideration $v$ and on the neighboring vertices $v_{j}$. (b) Angles $\alpha_{i}$ between the normal vector of the vertex $v$ and the normal vectors of the neighboring vertex $v_{j}$.

The clamp-histogram-based attribute vectors can distinguish one vertex from another, for two reasons. First, because the directions of normal vectors vary rapidly along the cortical surface, the neighboring vertices possess quite different attribute vectors, and this can easily be distinguished using clamp-histogram, as long as a surface patch of sufficient size is used. Second, the cortical surface is highly irregular and highly convoluted, which gives the distant vertices very different attribute vectors. As demonstrated in Fig 2, the clamp-histogram-based attribute vectors are quite distinctive for the displayed cortical surface patch. To reduce computation time, the 1-D clamphistogram, rather than the 2-D maps $[5,6]$, is used.

\subsection{Surface Warping}

The goal of surface warping is to further deform the volume-warped model surface $S^{\mathbf{W} \_ \text {Mmdl }}$ to the individual surface $S^{\text {Sub }}$, using an attribute-based correspondence matching method. For each vertex $v$ in the volume-warped model surface $S^{\mathbf{W}}$ Mmdl , we seek its correspondence in the target surface $S^{\text {Sub }}$. If the correspondence is successfully determined, the surface patch $P(v, r)$ around vertex $v$ will be deformed to the individual surface by a local transformation $T_{v}$. Therefore, the transformation $h_{2}$ in the second warping step is decomposed into a number of local transformations 
$\left\{T_{v}\right\}$, i.e., $h_{2}=\left\{T_{v}\right\}$. Mathematically, the surface warping procedure can be formulated as a procedure of minimizing the following energy function:

$$
E\left(h_{2}=\left\{T_{v}\right\}\right)=\sum_{v \in S^{\mathrm{W}}-\text { Mdl }}\left(w_{\text {Ext }} E_{v}^{\mathbf{E x t}}\left(T_{v}\right)+w_{\text {Int }} E_{v}^{\text {Int }}\left(T_{v}\right)\right)
$$

where $E_{v}^{\text {Ext }}\left(T_{v}\right)$ and $E_{v}^{\text {Int }}\left(T_{v}\right)$ are external and internal energy terms, defined for the vertex $v . w_{\text {Ext }}$ and $w_{\text {Int }}$ are weighting parameters.

The external energy term $E_{v}^{\text {Ext }}\left(T_{v}\right)$ measures the similarity of the surface patches in the model and the target, respectively. It requires that the attribute vector of vertex $v_{j}$ in the surface patch $P(v, r)$ be similar to that of its counterpart in the target surface, and also the normal direction on vertex $v_{j}$ be close to that on its counterpart. The mathematical definition is given as:

$$
E_{v}^{\mathrm{Ext}}\left(T_{v}\right)=\sum_{v_{j} \in P(v, r)}\left(w_{1}\left\|A\left(v_{j}\right)-A\left(m\left(T_{v}\left(v_{j}\right)\right)\right)\right\|+w_{2}\left\|n\left(v_{j}\right)-n\left(m\left(T_{v}\left(v_{j}\right)\right)\right)\right\|\right)
$$

where $m($.$) denotes the projection of a point to the closest vertex in the target surface,$ since the transformed vertex $T_{v}\left(v_{j}\right)$ is not necessary on the target vertex. $n\left(v_{j}\right)$ denotes the normal direction of $v_{j} \cdot w_{1}$ and $w_{2}$ are weighting parameters. The operation \|\|$\|$ calculates the magnitude of vector.

The internal energy term $E_{v}^{\text {Int }}\left(T_{v}\right)$ is designed to preserve the shape of the model surface during the deformation. As proposed in [4], a geometric attribute vector can be defined for each vertex $v$, which is actually a number of volumes of tetrahedrons, formed by the vertex $v$ and its three neighboring vertices in the different neighborhood layers. For example, $F(v)$ is a geometric attribute vector of the vertex $v$ in the volume-warped model surface $S^{\mathrm{w}-\mathrm{Mdl}}$, and $F\left(T_{v}(v)\right)$ is the geometric attribute vector of the vertex $T_{v}(v)$, deformed from $v$ by a local transformation $T_{v}$. Then, the internal energy function is defined as:

$$
E_{v}^{\text {Int }}\left(T_{v}\right)=\sum_{v_{j} \in P(v, r)}\left\|F\left(v_{j}\right)-F\left(T_{v}\left(v_{j}\right)\right)\right\| / \sum_{v_{j} \in P(v, r)} 1
$$

We use a greedy deformation algorithm to minimize energy function in Eq. (2). The adaptive-focus deformation strategy in [4] is also adopted here by firstly deforming the model vertices with the most distinctive attribute vectors to their counterparts in the individual, and then gradually involving vertices with less distinctive attributes. Notably, we deform the surface vertices within a small volume around each model vertex together, rather than deforming only one vertex. This avoids self-intersection of the deformed model surface and increases the chances of avoiding local minima. 


\section{Results}

We use both real brain images and images obtained via synthesized elastic warping, to demonstrate the performance of the proposed method. The synthesized images are obtained from an elastic warping algorithm as detailed in [3], applied to T1-weighted MR images segmented by the method described in [7]. For the synthesized images, we know the exact correspondences of vertices in their cortical surfaces. In this way, we can directly compare the registration errors that occur when using only the volumetric warping step, with those that occur when using the hybrid volumetric and surface warping method. As visually displayed in Fig 3, the registration result was improved by using a surface-based warping step. In Fig 3, the red-shaded area denotes a small registration error, while the blue-shaded area denotes a large registration error. The average registration error on this selected synthesized brain is reduced by $0.3 \mathrm{~mm}$ through use of the surface warping step. Fig 4 shows additional comparisons over four synthesized brain images, with Fig 4a showing the comparison on average registration errors, and Fig $4 \mathrm{~b}$ showing the comparison on maximal registration errors. By using the surface warping step, the average registration error is reduced by $0.2 \mathrm{~mm}$, and the average maximal error is reduced by $1.4 \mathrm{~mm}$. Notably, for those four synthesized brain images, the hybrid volumetric and surface warping method produced an average registration error of $0.92 \mathrm{~mm}$, and an average maximal registration error of $4.02 \mathrm{~mm}$.

We also tested our cortical surface registration algorithm on four real brain data sets. Since in the real data we don't know the correspondences among the cortical surfaces, we used the surface distance to measure the registration errors of our algorithm. Although this is not a direct validation method, it does evaluate how well our approach achieves its objective, i.e., to match two surfaces. We note attribute similarity, and not surface distance, was used as a criterion in the surface warping procedure. Therefore, surface distance is a somewhat independent evaluation criterion. By using the hybrid volumetric and surface warping method, the average distance of finally warped model surfaces to individual surfaces is $0.22 \mathrm{~mm}$, and the average distance of individual surfaces to the finally warped model surfaces is $0.30 \mathrm{~mm}$. Table 1 gives detailed distance measurements of four real brains. It can be seen that the volumetric warping step provided a good initialization, and the surface warping step further refined the warping results. This can be further confirmed by observing a color-coded map of distances of the finally warped model surface to an individual cortical surface in Fig 5. In Fig 6, we also visually indicate the positions where the significant registration errors were reduced by the surface warping step, as indicated by the yellow arrows. For most regions, the volumetric warping step has done a good job, and the surface warping step thus changed little.

\section{Conclusion}

This paper presented a hybrid volumetric and surface warping method for deformable registration of human cortical structures. The first step in the volumetric warping is 


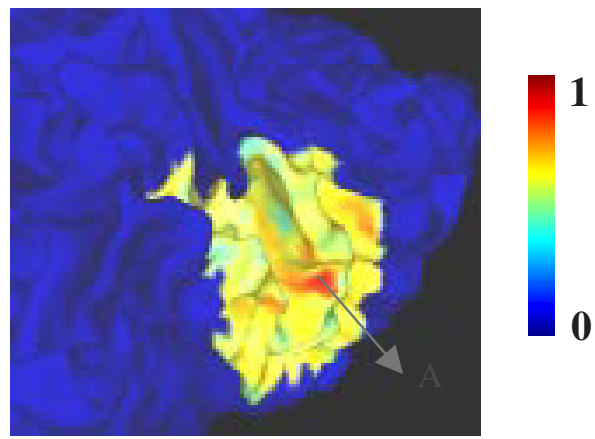

Fig. 2. Demonstration of attribute vectors' ability to distinguish vertices in the cortical surface. The degrees of similarity between the attribute vector of vertex A and the attribute vectors of vertices in its neighborhood are color-coded by a color bar on the right. Red represents high similarity, while blue denotes low similarity.

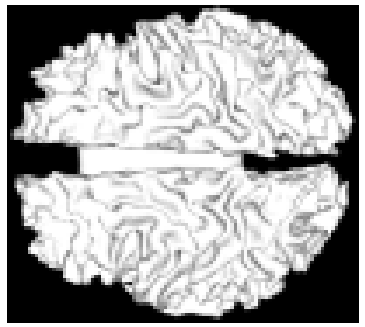

(a)

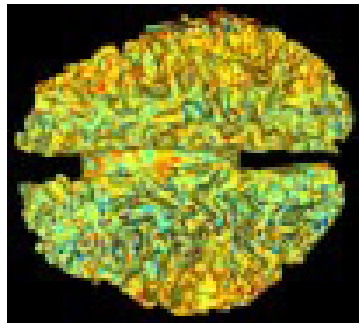

(b)

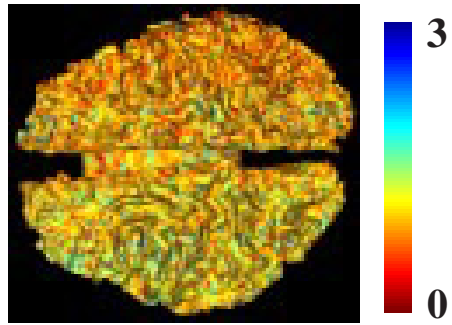

(c)

Fig. 3. Registration errors on a synthesized image. Registration errors are color-coded by a color bar on the right. The unit is mm. (a) Model surface. (b) Registration errors resulting from a volumetric warping step. (c) Registration errors resulting from a hybrid registration method.

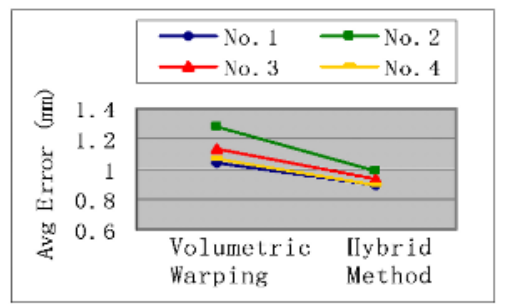

(a)

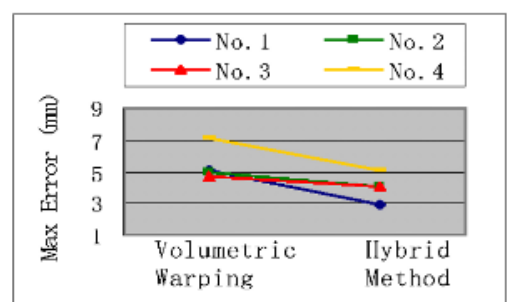

(b)

Fig. 4. Average and maximal registration errors on four synthesized images. (a) Average registration errors. (b) Maximal registration errors. It can be observed that the surface warping step improved the registration results obtained from the volumetric warping step. 


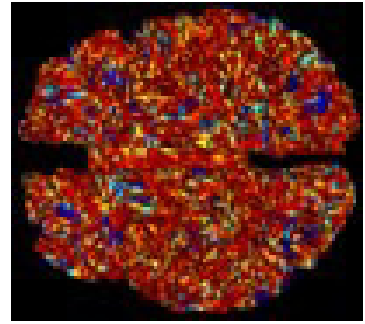

(a)

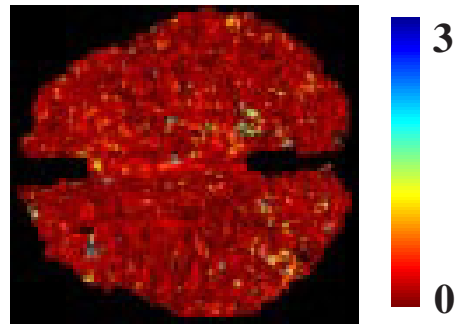

(b)

Fig. 5. Color-coded map of surface distances on a real brain. The unit is mm. (a) Surface distances resulting from a volumetric warping step. (b) Surface distances resulting from the hybrid volumetric and surface warping method.

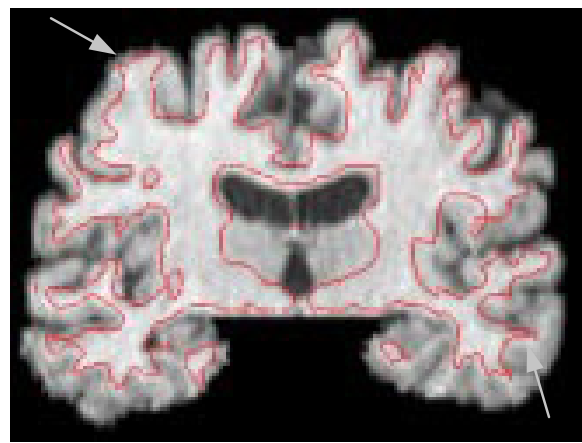

(a)

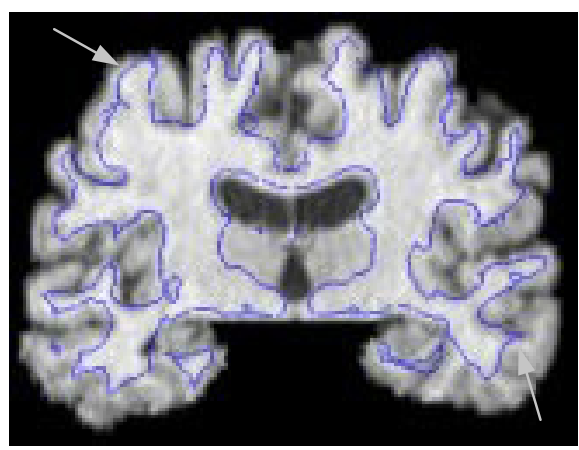

(c)

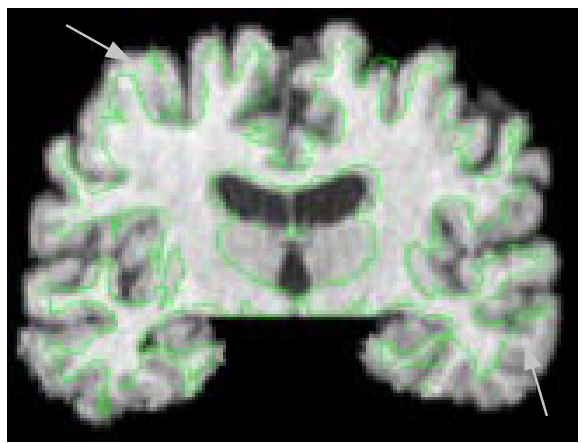

(b)

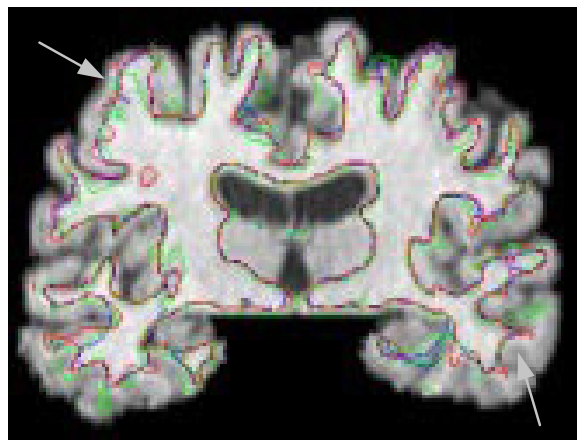

(d)

Fig. 6. Results of volumetric and hybrid methods. (a) Overlay of a representative brain slice and the cortical surface extracted from it (red curves). (b) The model's surface warped by a volumetric warping step (green curves). (c) The warped model surface further refined by a surface warping step (blue curves). (d) Overlay of the brain slice and above three surfaces. The yellow arrows indicate the positions where the registration results were significantly improved by the surface warping step. The underlying MR image is the same in (a-d). 
based on the HAMMER warping algorithm, and this first step greatly removes the variability existing between the cortical surfaces of the model and the individual, thereby providing a very good initialization for the sequent surface-based registration step. The clamp-histogram of the angles of the normal vectors is then used to provide a set of attributes that guide the surface warping. The performance of the proposed method was tested on both synthesized and real brain images. In the future, the proposed method will be further validated using a large set of real brain data, as well as using landmarks for which anatomical correspondence is known.

Table 1. Evaluation of registration performance on four real brain images. ' $M$ ' represents a finally warped model surface, and ' $S$ ' represents an individual cortical surface. 'M-S' denotes the distance from ' $M$ ' to 'S'. 'VolWarp' represents volumetric warping, and 'HybWarp' represents hybrid volumetric and surface warping. The average distance is provided.

\begin{tabular}{|r|c|c|c|c|}
\hline \multirow{2}{*}{ Subject } & \multicolumn{2}{|c|}{ M-S distance $(\mathrm{mm})$} & \multicolumn{2}{c|}{ S-M distance $(\mathrm{mm})$} \\
\cline { 2 - 5 } & VolWarp & HybWarp & VolWarp & HybWarp \\
\hline No.1 & 0.52 & 0.20 & 0.38 & 0.25 \\
\hline No.2 & 0.51 & 0.22 & 0.45 & 0.34 \\
\hline No.3 & 0.59 & 0.23 & 0.44 & 0.34 \\
\hline No.4 & 0.53 & 0.22 & 0.39 & 0.27 \\
\hline
\end{tabular}

\section{References}

1. J. Feldmar and N. Ayache, "Locally affine registration of free-form surfaces," in CVPR 1994, Seattle, WA, pp. 496-501, 1994.

2. Y. Wang, B. S. Peterson and L. H. Staib, "3D Brain surface matching based on geodesics and local geometry," Computer Vision and Image Understanding, 2003 (in press).

3. D. Shen and C. Davatzikos, "HAMMER: hierarchical attribute matching mechanism for elastic registration," IEEE Trans. on Medical Imaging, 21(11): 1421-1439, Nov. 2002.

4. D. Shen, E. H. Herskovits, and C. Davatzikos, "An adaptive-focus statistical shape model for segmentation and shape modeling of 3D brain structures," IEEE Transactions on Medical Imaging, 20(4): 257-270, April 2001.

5. A. E. Johnson and M. Hebert, "Using spin-Images for efficient multiple model recognition in cluttered 3-D scenes," IEEE Trans. on PAMI, 21(5): 433-449, 1999.

6. S. M. Yamany and A. A. Farag, "Surfacing signatures: an orientation independent freeform surface representation scheme for the purpose of objects registration and matching," IEEE Transactions on PAMI, 24(8): 1105-1120, 2002.

7. A. F. Goldszal, C. Davatzikos, D. L. Pham, M. X. H. Yan, R. N. Bryan, and S. M. Resnick, "An image-processing system for qualitative and quantitative volumetric analysis of brain images," Journal of Computer Assisted Tomography, 22(5):827-837, 1998. 